\title{
ANÁLISIS MORFOMÉTRICOS EN ARQUEOMALACOLOGÍA: IDENTIFICACIÓN DE ZONAS DE RECOLECTA EN CONCHALES DEL CANAL BEAGLE
}

ESTER VERDÚN C.

\section{RESUMEN}

Las costas del canal Beagle presentan una ocupación continuada de más de 7000 años, hasta la llegada de los marineros y colonos europeos en el s. XIX- XX. Por esta razón nos ha llegado información etnográfica sobre los grupos indígenas que habitaban la zona en ese momento. Según las crónicas, el canal Beagle estaba ocupado por la sociedad yamana. Este grupo tenía una economía basada en la cazapesca-recolección, con un consumo intensivo de los recursos costeros, principalmente moluscos. Esto se refleja en la gran concentración de conchales que se encuentran a lo largo de la costa.

El análisis morfométrico de las valvas de los moluscos puede aportar información sobre las características de la zona de captación de estos recursos, del radio de explotación y del tipo de gestión implementado. En este trabajo se presentan los resultados de la aplicación del estudio mofométrico en las especies Nacella deaurata, Nacella magellanica y Mytilus edulis de los yacimientos Túnel VII y Lanashuaia, correspondientes a la época de contacto. Se ha podido determinar una explotación intensiva, sin llegar a la sobreexplotación, de todo el gradiente intermareal.

PALABRAS CLAVE: arqueomalacología, conchales, Canal Beagle, yamana.

\section{MORPHOMETRIC ANALYSES IN ARCHAEOMALACOLOGY: IDENTIFICATION OF COLLECTION AREAS IN SHELL MIDDENS OF BEAGLE CHANNEL}

\begin{abstract}
The Beagle Channel coasts have been continuously occupied during the last 7000 years until the arrival of European sailors and settlers at $19^{\text {th }}-20^{\text {th }}$ centuries. That is why we are nowadays able to get ethnographic information about native people who lived there during that period. Following the chronicles, the Beagle Channel was occupied by yamana society. This group had an economic system based on hunting- fishing- gathering. There was an intensive consumption of coastal resources, especially molluscs. It is proved by the huge concentration of shell middens along the coast.

The morphometric analysis of the shells affords information about the characteristics of the

Laboratori d'Arqueozoologia, Universitat Autònoma de Barcelona; Grupos de investigación AGREST (Generalitat de Catalunya), GASA (UAB); Campus UAB, Edifici M, 08193, Bellaterra, Catalunya, España; estervc@gmail.com
\end{abstract}


collection area, about the area of exploitation and the management pattern. This study shows the results obtained by the application of morphometrics to Nacella deaurata, Nacella magellanica and Mytilus edulis species from Túnel VII and Lanashuaia sites. Both sites correspond to the contact period. An intensive exploitation of molluscs has been determined. However, an overexploitation of molluscs along the intertidal area never existed.

KEY WORDS: archaeomalacology, Shell middens, Beagle Channel, yamana.

\section{INTRODUCCIÓN}

La costa del canal Beagle presenta un contexto arqueológico excepcional por la alta densidad de yacimientos y por el marco cronológico que abarcan. Se documenta una ocupación ininterrumpida desde 7800 BP hasta la llegada de colonos y marineros europeos en el s. XIX- inicios del s. XX (Orquera \& Piana, 2009). El contacto con la sociedad occidental propició la existencia de abundante información etnográfica (grabados, fotografías y crónicas) (p. ej. Hyades \& Deniker, 1891; Bridges, 1975; Gusinde, 1986). Durante todo el periodo la costa fue habitada por grupos cazadores-recolectores-pescadores, lo que queda constatado en los últimos tiempos por los testimonios etnográficos. Según éstos, era la sociedad yamana la que habitaba esta zona en este momento.

La mayoría de los yacimientos son conchales, lo que indica la intensa explotación de los recursos costeros por parte de los grupos que vivían en esta zona (Orquera, 1999; Verdún, 2006; Piana \& Orquera, 2010). Están formados por una alta densidad de moluscos- sobre todo mejillones (Mytilus edulis Hupé 1854) y lapas (Nacella deaurata (Gmelin, 1791) y Nacella magellanica (Gmelin, 1790)- y en general, presentan una sucesión de ocupaciones cortas, lo que también apoyan las crónicas etnográficas (Gusinde, 1986). Se cree que la razón de la brevedad de las ocupaciones puede ser la explotación de recursos "no-móviles" cercanos al asentamiento, como los bancos de moluscos (Mameli \& Estévez, 2004; Verdún, 2014a). No obstante, la información sobre su gestión aun es escasa.

Además de la información paleoeconómica, los restos arqueomalacológicos son una fuente importante de información paleoecológica. Esta se obtiene a partir de la composición taxonómica del conjunto (asociaciones de especies), la composición química de la valva y de su morfología (p. ej. Seed, 1980; Claassen, 1998; Cabral \& da Silva, 2003; Rick et al. 2005; Hallmann et al. 2009). La morfología de las valvas está directamente relacionada con las características de la costa en la que estos animales han vivido, por lo que su estudio puede aportar información sobre las zonas de captación e indirectamente sobre el tipo de gestión implementado (Cabral \& da Silva, 2003; Campbell, 2008; Cabral, 2010).

Son muchos los factores naturales que pueden afectar la morfología de las valvas. Por un lado están las condiciones ambientales (la latitud, la profundidad, el nivel de la costa, el nivel de la marea, las corrientes, las turbulencias del agua, la exposición a las olas, la densidad de la composición de los bancos, el tipo de sustrato y el tipo de sedimento). Por otro lado están los factores fisiológicos específicos ya que el tipo y el grado de alteración de las valvas puede variar entre especies y por la zona donde se encuentren (Seed, 1968, 1980; Behrens Yamada \& Peters, 1988; Gaspar et al. 2002). Estas condiciones pueden afectar directamente al grosor, anchura, longitud, altura y peso de la valva (Gaspar et al. 2002; Morriconi, 2005).

Este trabajo tiene el objetivo de contribuir a reconstruir las estrategias socio-económicas de la sociedad yamana, ofreciendo nuevos datos sobre modelos de gestión de los recursos costeros, principalmente de los moluscos. Especialmente en el contexto arqueológico que presenta el canal Beagle, los análisis morfométricos en valvas pueden permitir caracterizar las zonas de recolecta en los periodos breves de ocupación e identificar el radio de explotación de los moluscos por parte de los grupos que habitaron los yacimientos Túnel VII y Lanashuaia. Este trabajo pretende ofrecer un ejemplo sobre la potencialidad de la aplicación de los estudios morfométricos y sobre la información que pueden aportar en el estudio de las sociedades 

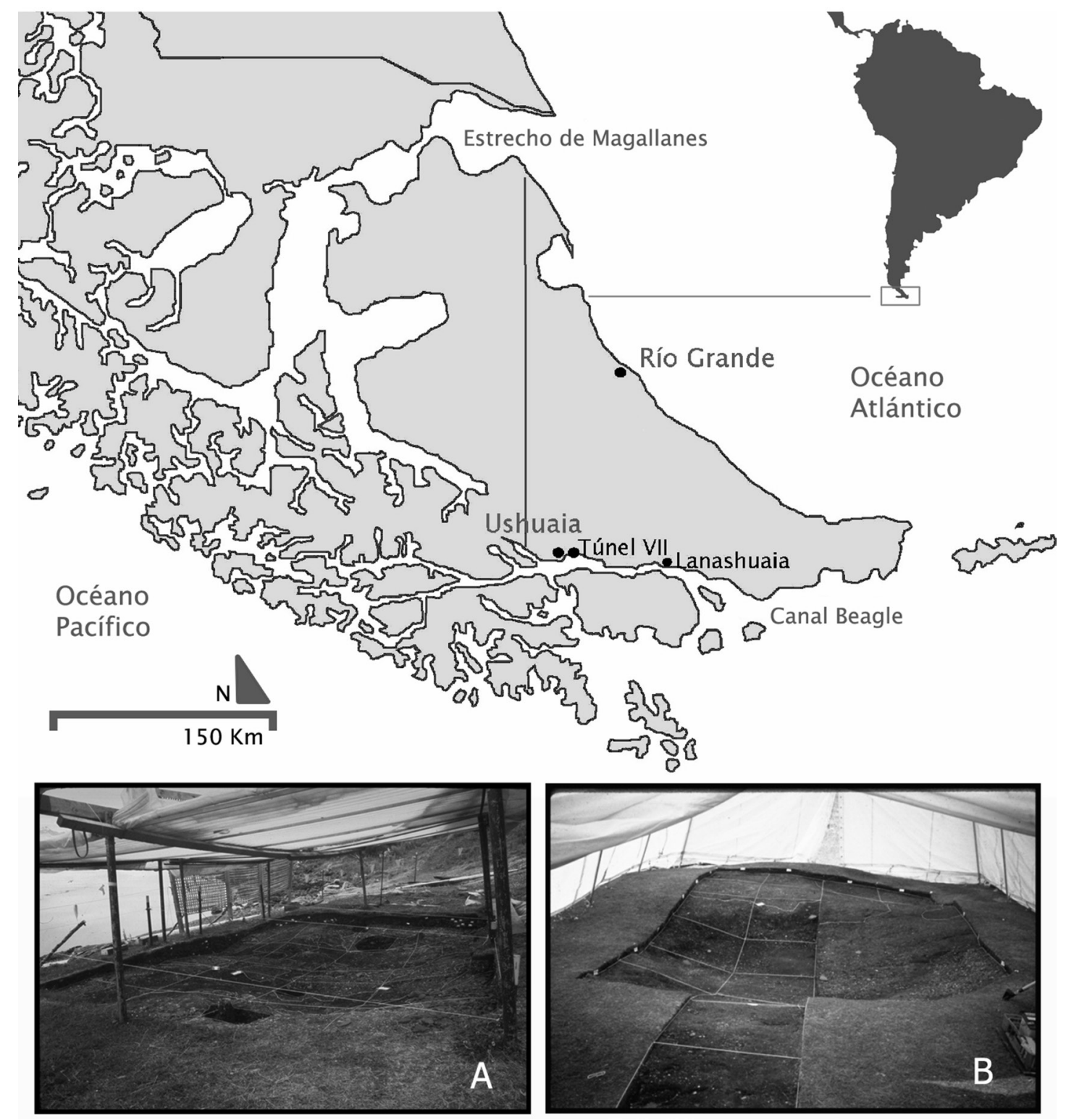

Fig. 1. Localización e imágenes de Túnel VII (A) y Lanashuaia (B).

del pasado. La aplicación de estos estudios en otros contextos arqueológicos puede aportar información relevante para caracterizar las zonas de captación y gestión de estos recursos.

\section{LOS CONCHALES TÚNEL VII Y LANASHUAIA}

Túnel VII y Lanashuaia son dos conchales detalladamente estudiados que se encuentran en la costa norte del canal (Fig. 1 y 2). Los dos yacimientos presentan una estructura similar, como la mayoría de los conchales de la zona (Piana \& Orquera, 2010). Están formados por una estructura circular de c. $3 \mathrm{~m}$ de diámetro con una parte central semi-excavada, donde se ubicaban las cabañas, circundada por una "estructura" más elevada a modo de zócalo de protección contra el viento y el agua. Es en esta parte donde se produce la mayor concentración de valvas, además de otros residuos 
(Verdún, 2010). La estructura estratigráfica de los conchales de la zona es muy compleja. Están formados por subunidades estratigráficas (unidades mínimas de depósito estratigráfico) y representan
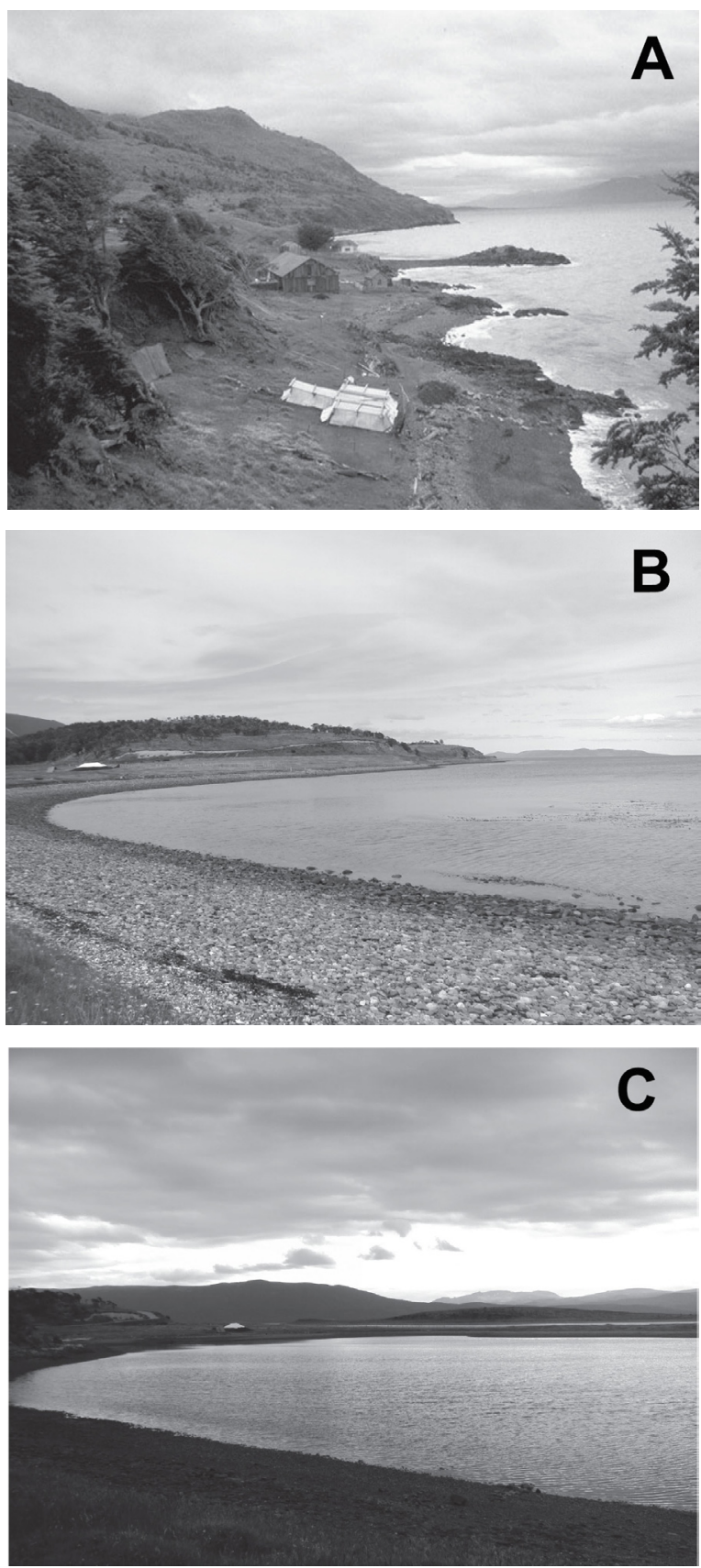

Fig. 2. Entorno en el que se encuentran los sitios estudiados Túnel VII (A) en la costa del canal Beagle y Lanashuaia, entre las bahías Cambaceres exterior (B) e interior (C). (Fotografía A, realizada por J. Estévez) episodios singulares de acumulación (Orquera \& Piana, 1992). Los paquetes de subunidades forman los episodios de ocupación.

Túnel VII está formado por 10 episodios de ocupación que se dataron por dendrocronología entre el s. XVIII y finales del s. XIX (Estévez \& Vila, 1995, 2006). Se estimó la cantidad total de moluscos en los episodios de ocupación B, C y D. La ocupación B tenía 15.508 individuos; la C, 11.816 y la D, 68.264. Las especies mayoritarias son los mejillones, que representan el $89-92 \%$ y las lapas, que representan entre 2 y $7 \%$. Los análisis isotópicos realizados en Nacella magellanica de los episodios $\mathrm{G}, \mathrm{H}$ y $\mathrm{J}$ indican que el asentamiento estuvo habitado en distintos momentos del año. Los valores de $\mathrm{G}$ muestran una recolección de otoño/invierno, $\mathrm{H}$ corresponde a primavera/ verano y $\mathrm{J}$ indica una recolección mayoritaria en otoño (Colonese et al. 2011).

Lanashuaia se encuentra a c. $60 \mathrm{~km}$ de Túnel VII, en el istmo que queda entre las bahías Cambaceres interior y exterior. Las dos bahías presentan características ecológicas distintas ya que la interior es más calma y protegida del oleaje que la exterior (Fig. 2). Cronológicamente Lanashuaia corresponde a la época de contacto con los europeos (Piana et al. 2000; Álvarez et al. 2009; Briz et al. 2009). Forma parte de un conjunto de yacimientos de características similares dispuestos siguiendo la línea de costa. Esta disposición lineal de las estructuras y la recuperación de huesos de ballena minke en la base del sitio (Balaenoptera acutorostrata), en combinación con lo descrito en las crónicas etnográficas llevó a plantear la hipótesis de que podría tratarse de un momento de agregación de distintas unidades familiares (Piana et al. 2000; Álvarez et al. 2009; Briz et al. 2009). Varios testimonios etnográficos describían eventos de agrupación cuando una ballena varaba en la costa con el fin de consumirla (p. ej. Gusinde, 1986). Estratigráficamente se detectó un solo momento de ocupación que corresponde con la hipótesis de ocupación del sitio. Los análisis isotópicos en Nacella magellanica indican que estos individuos fueron capturados mayoritariamente en invierno (Colonese et al. 2011). En Lanashuaia se estimó una cantidad de 345.165 moluscos, de los que el $74 \%$ son mejillones y el $9 \%$ son lapas (Verdún, 2014a). 


\section{CONSIDERACIONES ECOLÓGICAS DE LAS ESPECIES ESTUDIADAS}

Los análisis morfométricos se aplicaron a mejillones (Mytilus edulis) y lapas (Nacella deaurata y Nacella magellanica) por ser las especies más importantes como alimento para la sociedad yamana y porque se disponía de información morfométrica previa para el canal Beagle. Además son varios los estudios realizados en lapas y mejillones en otras zonas que demuestran la influencia de las condiciones ecológicas en la morfología de estos taxones (p. ej. Seed, 1968, 1980; Cabral \& da Silva, 2003; Campbell, 2008, 2014).

\section{Nacella deaurata y Nacella magellanica}

Estas dos especies se encuentran en la zona intermareal. El régimen de marea es de desigualdad diurna: hay dos bajamares diarias, una más baja que la otra; de las dos pleamares, una es más alta que la otra (Zaixso et al. 1978). Nacella deaurata se encuentra en la zona inferior de la franja eulitoral y sublitoral, mientras que Nacella magellanica ocupa la zona eulitoral superior y media (según la nomenclatura de Russell (1991) y Zaixso et al. (1978) usada por Morriconi y Calvo (1993) y Morriconi (1999). Según Morriconi (2005), en el canal Beagle las dos especies están directamente afectadas por la acción eólica y de las olas, a lo que responden con distintos sistemas adaptativos que quedan reflejados en la morfología de la valva. Los individuos de Nacella deaurata que habitan costas expuestas, con mayor acción de las olas y vientos constantes, presentan valvas significativamente más altas y pesadas respecto a su longitud que los individuos de costas protegidas. Dado que esta especie generalmente ocupa zonas cubiertas por el agua, Morriconi y Calvo (1993) relacionan esta variación en la morfología valvar con el grado de exposición al oleaje, siendo un mecanismo de defensa contra el desprendimiento. Estos animales se adhieren más al sustrato, propiciando la retracción del borde del manto y disminuyendo el crecimiento periférico. Esto causa un mayor crecimiento en altura de las valvas y un mayor engrosamiento de éstas, aumentando su peso (Morriconi \& Calvo, 1993; Morriconi, 2005).
Los individuos de Nacella magellanica de costas expuestas al oleaje y a los vientos dominantes, al contrario que $N$. deaurata, presentan valvas más bajas que los individuos de costas más protegidas. Por la zona que ocupan en el intermareal, los individuos de esta especie pasan mucho tiempo fuera del agua. En condiciones normales de marea, estos animales están expuestos al aire de 3 a 5 horas dos veces al día (Malanga et al. 2005). En marea de cuadratura, $N$. magellanica queda emergida 9,5 horas diarias y en marea de sicigia, más de 11 horas (Morriconi, 2005). Por ello, los ejemplares de esta especie que habitan zonas protegidas se adhieren al sustrato para evitar la desecación. Esto provoca, como en el caso anterior, una retracción del borde del manto, la disminución del crecimiento periférico y el aumento del crecimiento en altura de la valva (Morriconi, 2005).

\section{Mytilus edulis}

Mytilus edulis es la especie más abundante en la franja media e inferior eulitoral de las costas del canal Beagle (Zaixso et al. 1978; Silva, 1996). Un estudio exhaustivo centrado en mejillones de las costas de la Provincia de Buenos Aires (Bala, 1989) demuestra que existe una relación entre la morfología de las valvas y la profundidad a la que estas se encuentran. Los ejemplares que están constantemente sumergidos soportan condiciones ambientales más estables, a diferencia de los individuos de zonas superiores (sometidos a constantes cambios de temperatura, salinidad y grados de insolación). Para las costas del canal Beagle existen estudios preliminares que llegan a conclusiones similares. Demuestran que ejemplares provenientes de criaderos en longline, suspendidos en zonas semiexpuestas del canal Beagle, presentan valvas proporcionalmente más altas, más ligeras y menos anchas que los ejemplares provenientes de la zona intermareal (Silva \& Calvo, 1995; Silva, 1996). Éstos últimos están constantemente expuestos a la desecación, cambios en la temperatura y salinidad, acción eólica y de las olas o a la imposibilidad de alimentarse mientras están emergidos. Los individuos provenientes de franjas inferiores del intermareal no están sometidos a este estrés constante. Los ejemplares de zonas más estables tendían a tener proporcionalmente valvas 
más largas y ligeras, mientras los ejemplares de zonas de condiciones variables presentaban valvas proporcionalmente más gruesas y anchas (Silva, 1996).

\section{MATERIAL Y MÉTODOS: ESTUDIO DE LOS RESTOS ARQUEOLÓGICOS}

Los restos arqueológicos analizados provienen de muestras sedimentarias de cada una de las subunidades estratigráficas que componen Túnel VII y Lanashuaia. Se trata de muestras de $4 \mathrm{dm}^{3}$ de sedimento, obtenidas en horizontal y representativas de la composición del total de cada subunidad (Orquera \& Piana, 2000). A continuación se explica como se realizó el estudio en cada una de las especies.

Nacella deaurata y Nacella magellanica

En base a la información biológica y ecológica de estas especies (Morriconi \& Calvo, 1993; Malanga et al. 2005; Morriconi, 2005) para obtener información sobre la zona de captura de las lapas arqueológicas se midieron los ejemplares de las dos especies con un calibre vernier digital. Se obtuvo la longitud máxima (Lt) y la altura (Al) ya que, según los estudios expuestos (Morriconi \& Calvo, 1993; Morriconi, 2005), son las medidas de las valvas que varían en función de las condiciones ecológicas locales (Fig. 3). Para N. deaurata no se tuvo en cuenta la variable "peso" por ofrecer muchas críticas en la aplicación al estudio de los moluscos arqueológicos (p. ej. Mason et al. 1998). La caracterización morfométrica se realizó mediante el cálculo de un índice a partir de estas variables ( $\mathrm{Al} / \mathrm{Lt})$, siguiendo la propuesta de distintos autores (Morriconi \& Calvo, 1993; Cabral \& da Silva, 2003; Campbell, 2008). Este indice permitía efectuar comparaciones entre los ejemplares.

Se recolectaron ejemplares modernos de zonas cercanas a los sitios arqueológicos para realizar comparaciones con los individuos arqueológicos. Estos individuos fueron obtenidos de la zona superior/media eulitoral del intermareal, fueron medidos y se calculó el índice Al/Lt. Todos los datos obtenidos fueron sometidos a análisis estadísticos con el programa PAST (tests de normalidad, Shapiro-Wilk, y de varianza, one-way ANOVA o Kruskall-Wallis) (Hammer et al. 2001).

\section{Mytilus edulis}

La caracterización morfológica de los ejemplares se realizó a través del cálculo del índice obtenido entre la anchura (An) y la longitud de las valvas (Lt) (Fig. 3). Los datos obtenidos de los ejemplares arqueológicos se compararon con los índices de ejemplares modernos obtenidos en zonas próximas a los yacimientos arqueológicos. Los datos se analizaron estadísticamente con

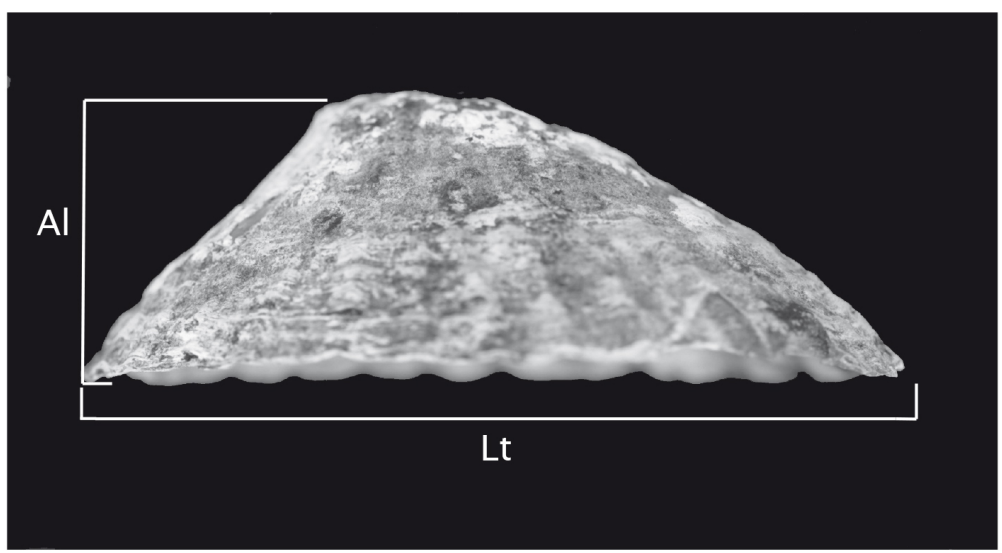

Fig. 3. Medidas tomadas en Nacella deaurata, Nacella magellanica (izquierda) y Mytilus edulis (derecha).

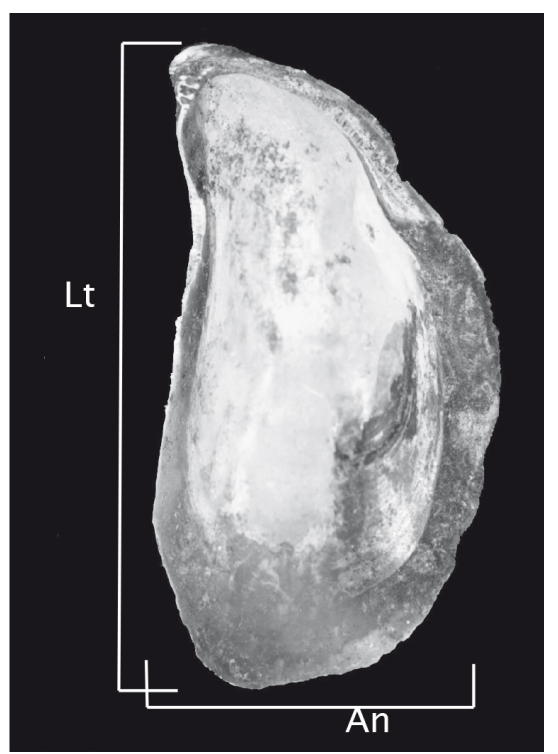


el programa PAST (Hammer et al. 2001). Se realizaron tests de normalidad (Shapiro-Wilk) y tests de varianza (one-way ANOVA o Kruskal-Wallis).

\section{RESULTADOS}

En general, el número de individuos analizado en los dos yacimientos es bajo (especialmente en el tratamiento por ocupaciones en Túnel VII) debido a que se obtuvieron de las muestras sedimentarias y a que los restos presentan un alto grado de fractura.

\section{Túnel VII}

Se analizó el material proveniente de las ocupaciones B, D, E, F, G, H, J (B es la más antigua) (Estévez \& Vila, 2006). Los datos se trataron por

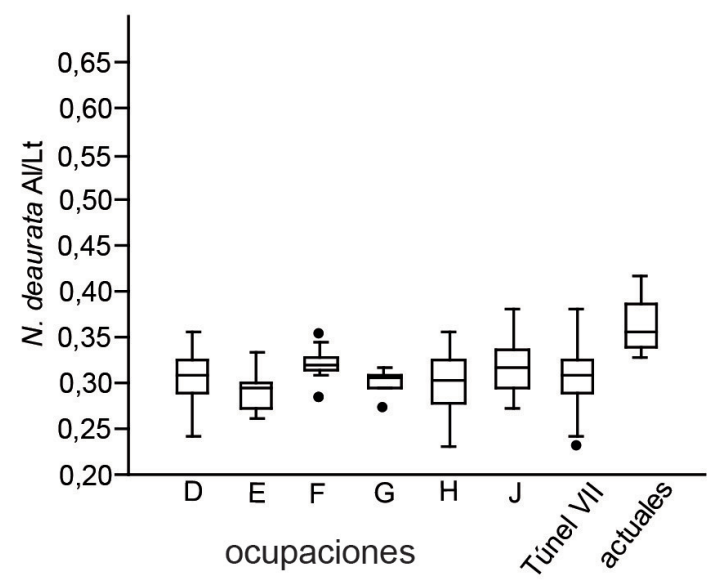

ocupaciones pero también se analizaron en su totalidad (en los gráficos y tablas se presenta con la etiqueta "Túnel VII"). Dado que el número de ejemplares medidos es bajo, la agrupación de datos ofrecía la posibilidad de caracterizar el sitio en su conjunto frente a los datos aportados por los ejemplares actuales.

Los índices obtenidos de los ejemplares de Nacella deaurata de todo el sitio de Túnel VII están comprendidos entre 0,23 y 0,38 , siendo la media de 0,31 . La variabilidad en los datos obtenidos es escasa (St. Dev.=0,03) (Tabla 1 y Fig. 4). La ocupación $\mathrm{E}$ es la que presenta ejemplares con un indice medio más bajo $(0,29)$. Las ocupaciones con valores medios más altos son $\mathrm{F}$ y J $(0,32)$. El test de varianza indica que existen diferencias significativas entre los valores obtenidos en las distintas ocupaciones (one-way ANOVA: $p=0,03574$ ) (tabla

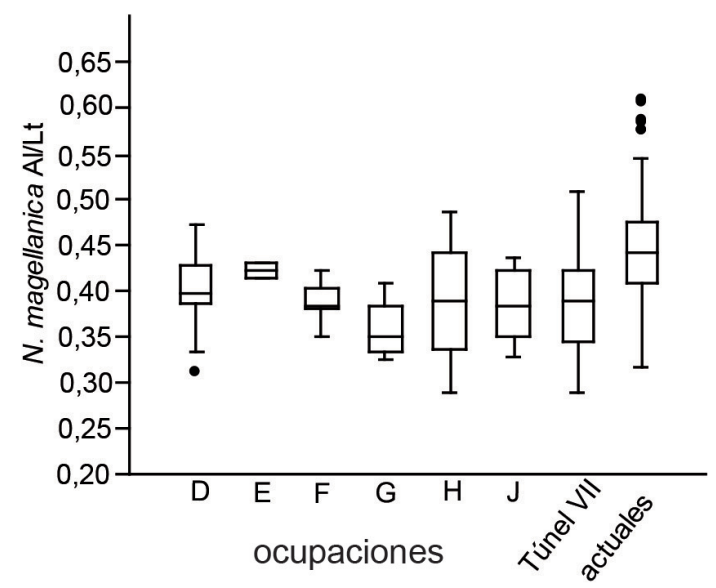

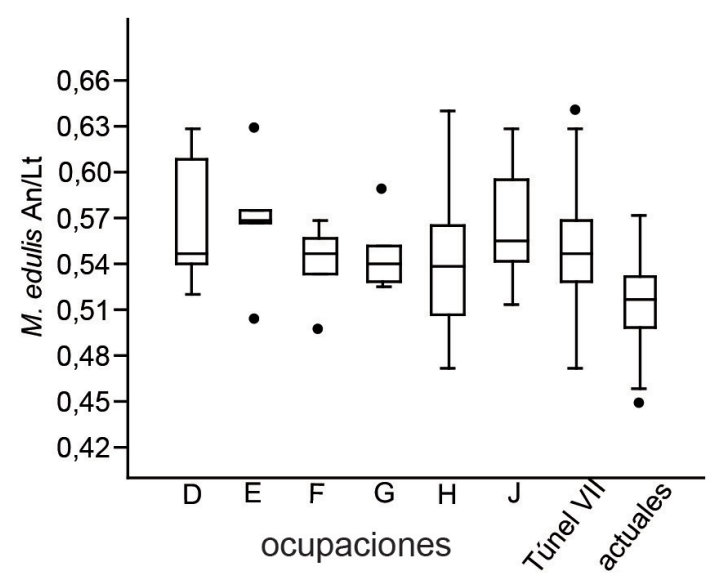

Fig. 4. Gráfico de cajas del índice (Al/Lt) de N. deaurata, N. magellanica y Mytilus edulis arqueológicos y actuales de Túnel VII. La etiqueta Túnel VII corresponde a la totalidad de los datos del sitio. 
1). Los ejemplares actuales presentan un índice medio de 0,36 (tabla 1, Fig. 4). Se compararon los valores obtenidos de los ejemplares de Túnel VII con los de los ejemplares actuales mediante el test de varianza y se comprobó la existencia de diferencias significativas entre las dos poblaciones (one-way ANOVA: $p=2,126 \mathrm{E}-08$ ).

Los individuos de Nacella magellanica presentan valores comprendidos entre 0,29 y
0,51 , con una media de 0,39 . La variabilidad en los datos obtenidos es superior a la que presenta $N$. deaurata (St. Dev. = 0,05) (Fig. 4). Las ocupaciones $\mathrm{B}$ y $\mathrm{E}$ son las que presentan valores medios más altos, pero debido al escaso número de ejemplares de cada una, este resultado puede estar sesgado. La ocupación D presenta resultados más fiables, alcanzando una media de 0,40 y la ocupación $G$ es la que presenta un índice medio más bajo $(0,36)$.

Tabla 1. Medidas e índices de Nacella deaurata, Nacella magellanica y Mytilus edulis de Túnel VII,

Lanashuaia y actuales (Estancia Túnel, Cambaceres interior y Cambaceres exterior). Entre paréntesis se muestra el número de individuos que forman la muestra.

\begin{tabular}{|c|c|c|c|c|c|c|c|c|c|}
\hline \multirow[b]{3}{*}{ TÚNEL VII } & \multicolumn{6}{|c|}{ Gasterópodos } & \multirow{2}{*}{\multicolumn{3}{|c|}{$\frac{\text { Bivalvos }}{\text { Mytilus edulis }}$}} \\
\hline & \multicolumn{3}{|c|}{ N. deaurata } & \multicolumn{3}{|c|}{ N. magellanica } & & & \\
\hline & $\mathrm{Lt}$ & $\mathrm{Al}$ & $\mathrm{Al} / \mathrm{Lt}$ & Lt & $\mathrm{Al}$ & $\mathrm{Al} / \mathrm{Lt}$ & Lt & An & $\mathrm{An} / \mathrm{Lt}$ \\
\hline \multirow{2}{*}{ B } & 29,3 & 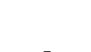 & & 33,1 & 16,8 & 0,51 & 28,2 & 15,9 & 0,56 \\
\hline & (1) & & & (1) & (1) & (1) & (1) & (1) & (1) \\
\hline \multirow{2}{*}{ D } & 33,7 & 10,2 & 0,31 & 32,7 & 13,2 & 0,4 & 29,7 & 17,5 & 0,57 \\
\hline & (20) & (20) & (18) & $(12)$ & $(11)$ & (10) & (10) & (10) & (8) \\
\hline \multirow{2}{*}{ E } & 29 & 8,4 & 0,29 & 32 & 13,5 & 0,42 & 25,8 & 12,3 & 0,57 \\
\hline & (10) & (9) & (9) & (2) & (2) & (2) & (6) & (5) & (5) \\
\hline \multirow{2}{*}{$\mathrm{F}$} & 31,4 & 10,1 & 0,32 & 24,4 & 9,5 & 0,39 & 29 & 16,5 & 0,54 \\
\hline & (24) & (17) & (17) & (5) & (5) & (5) & (7) & (6) & (6) \\
\hline \multirow{2}{*}{ G } & 32,8 & 9,7 & 0,3 & 32,3 & 11,4 & 0,36 & 28,8 & 14,5 & 0,55 \\
\hline & $(11)$ & (7) & (6) & (8) & (7) & (6) & (8) & (7) & (6) \\
\hline \multirow{2}{*}{$\mathrm{H}$} & 33,7 & 10,1 & 0,3 & 33,4 & 12,9 & 0,39 & 35,8 & 18,7 & 0,54 \\
\hline & $(30)$ & (27) & $(26)$ & $(15)$ & $(15)$ & $(15)$ & (26) & (25) & (23) \\
\hline \multirow{2}{*}{$J$} & 33,7 & 10,8 & 0,32 & 33,2 & 12,9 & 0,38 & 30,2 & 16,6 & 0,57 \\
\hline & $(20)$ & (20) & (19) & $(12)$ & (13) & $(11)$ & (12) & $(10)$ & (10) \\
\hline \multirow{2}{*}{ Total } & 32,7 & 10,1 & 0,31 & 32,2 & 12,5 & 0,39 & 31,5 & 17 & 0,55 \\
\hline & (116) & (100) & (96) & $(55)$ & $(54)$ & $(50)$ & (70) & (64) & (59) \\
\hline \multirow{2}{*}{ Estancia Túnel } & 41 & 15 & 0,36 & 43 & 19,4 & 0,44 & 55 & 28 & 0,52 \\
\hline & (10) & (10) & (10) & (113) & (113) & (113) & $(51)$ & (51) & (51) \\
\hline \multirow{2}{*}{ LANASHUAIA } & 37,9 & 11,8 & 0,32 & 41,9 & 15,6 & 0,37 & 35,3 & 20,1 & 0,56 \\
\hline & (39) & (49) & (35) & $(67)$ & $(68)$ & (57) & (97) & (105) & (72) \\
\hline \multirow[t]{2}{*}{ Cambaceres interior } & - & - & - & - & - & - & 58,7 & 28,7 & 0,49 \\
\hline & & & & & & & (87) & $(87)$ & (87) \\
\hline \multirow{2}{*}{ Cambaceres exterior } & 43,3 & 16,8 & 0,39 & 50,2 & 26 & 0,51 & 51,9 & 27,5 & 0,54 \\
\hline & (17) & (17) & $(17)$ & $(22)$ & $(22)$ & $(22)$ & (86) & (86) & $(86)$ \\
\hline
\end{tabular}



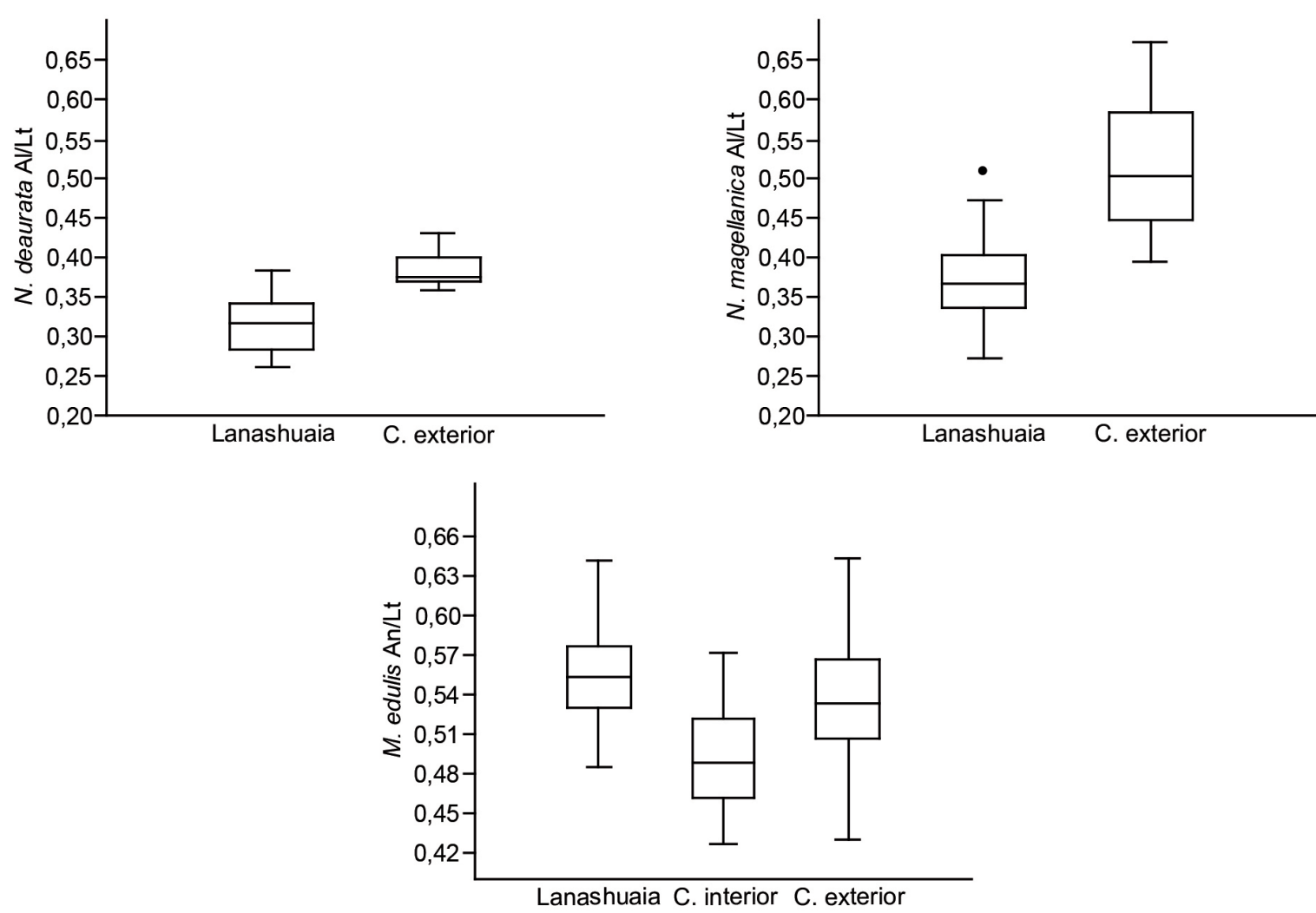

Fig. 5. Gráfico de cajas del índice (Al/Lt) de N. deaurata, N. magellanica y (An/Lt) de Mytilus edulis arqueológicos y actuales de Lanashuaia.

A diferencia de la dinámica observada en $N$. deaurata, el test de varianza no indica diferencias significativas entre las ocupaciones (KruskalWallis: $p=0,5907)$. En la aplicación del test se han excluido las ocupaciones B y E, por el bajo número de ejemplares que presentan. Las muestras actuales presentan un valor medio de 0,44. El test de varianza indica diferencias significativas entre la población arqueológica y la actual (Kruskal-Wallis: $\mathrm{p}=2,359 \mathrm{E}-08$ ).

Los ejemplares de Mytilus edulis presentan valores comprendidos entre 0,47 y 0,64 , con una media de 0,55 (Tabla 1). La variabilidad que presentan los datos no es elevada (St. Dev. $=0,04$ ). Las ocupaciones $\mathrm{D}, \mathrm{E}$ y J presentan el índice medio más alto $(0,57)$, mientras que las ocupaciones $\mathrm{F}$ y $\mathrm{H}$ presentan el más bajo $(0,54)$. No existen diferencias significativas entre las distintas ocupaciones (oneway ANOVA: $p=0,37)$. Para realizar este test se ha excluido la ocupación $\mathrm{B}$, dado que solo presenta un resultado.
Los ejemplares modernos presentan un índice medio de 0,52 (Tabla 1, Fig. 4). La comparación entre estos datos y los obtenidos de los individuos arqueológicos indica que existen diferencias significativas entre las dos poblaciones (Kruskal-Wallis: $p=7,268 \mathrm{E}-07$ ).

\section{Lanashuaia}

Lanashuaia presenta un solo momento de ocupación, por lo que el material se analizó en su conjunto. Los ejemplares actuales de Nacella deaurata y Nacella magellanica se obtuvieron de la bahía Cambaceres exterior ya que actualmente no hay lapas en la interior. Los mitílidos se obtuvieron en las dos bahías.

Los individuos de Nacella deaurata presentan un índice medio de 0,32, comprendido entre el valor mínimo de 0,26 y el máximo de 0,38 (tabla 1 y Fig. 5). Los datos obtenidos son bastante homogéneos (St. Dev.=0,03). El índice 
medio obtenido de los ejemplares actuales es de 0,39. La comparación de estos datos con los de Nacella deaurata de Lanashuaia indica que existen diferencias significativas entre las dos poblaciones (one-way ANOVA: $\mathrm{p}=1,517 \mathrm{E}-10$ ).

Los individuos de Nacella magellanica tienen índices comprendidos entre 0,27 y 0,51, con una media de 0,37. La desviación estándar indica que se trata de una población sin variabilidad elevada (St. Dev.= 0,04). Los ejemplares modernos de la costa de la bahía Cambaceres exterior presentan una media de 0,51 (tabla 1). La comparación entre la población arqueológica y la moderna indica que existen diferencias significativas (Kruskal-Wallis: $p=$ 5,225E-10).

Los ejemplares de Mytilus edulis presentan un índice medio de 0,56, comprendido entre 0,48 y 0,64 . No existe mucha variabilidad entre los datos (St. Dev.= 0,04). Los ejemplares modernos de la bahía Cambaceres interior presentan una media de 0,49 y los de la exterior, de 0,54. Aunque en los gráficos de cajas la población de Lanashuaia presenta similitudes con la población moderna de la Cambaceres exterior (Fig. 5), el test de varianza no es conclusivo ya que se detectaron diferencias significativas con los ejemplares obtenidos en las dos bahías (Cambaceres interior: one-way ANOVA: $p=8,143 \mathrm{E}-23$; Cambaceres exterior: one-way ANOVA: $p=0,0008352$ ).

\section{DISCUSIÓN}

Según Morriconi y Calvo (1993), el factor más importante que causa los cambios en la morfología de la valva de Nacella deaurata en el canal Beagle es la protección contra el desprendimiento por acción del oleaje. Los índices (Al/Lt) obtenidos para esta especie en Túnel VII indican que hay variaciones significativas entre las distintas ocupaciones, lo que, según los mismos autores y otros casos similares (p. ej. Cabral \& da Silva, 2003), podría indicar una explotación de zonas de recolecta con distinto grado de acción de las olas. Las ocupaciones $\mathrm{F}, \mathrm{J}$ y $\mathrm{E}$ son las que presentan valvas más altas y bajas respectivamente. Los valores de $\mathrm{F}$ y $\mathrm{J}$ indicarían una proveniencia mayoritaria de zonas más expuestas a la acción del oleaje que los ejemplares de $\mathrm{E}$.

Aunque el número de ejemplares analizado es bajo, existe una diferencia significativa entre los ejemplares arqueológicos de $N$. deaurata y los actuales: la población actual presenta valvas más altas (indices más altos), lo que indica una recolección en zonas más expuestas al oleaje que la población arqueológica. Los grupos que recolectaron estos animales en el pasado habrían obtenido las lapas en zonas con baja acción del oleaje (Morriconi \& Calvo, 1993; Morriconi, 2005). Esto podría indicar o bien una recolección mayoritaria en zonas más profundas del intermareal que los ejemplares modernos, o bien una recolección en zonas más estables, sin mucha acción del oleaje. Por otro lado, el intervalo de datos representado por los ejemplares arqueológicos ofrece más variabilidad que los modernos, lo que coincide con una mayor variabilidad en la zona de obtención.

Para Nacella magellanica, el factor preponderante en la variación morfológica de las valvas es la protección contra la desecación (Morriconi, 2005). Los datos de esta especie no presentan diferencias significativas entre ocupaciones, lo que sugiere una explotación de zonas con características similares en todas las ocupaciones. Aunque la variabilidad en los datos es escasa, se podría sugerir una obtención en zonas con distinto grado de afectación por las olas de manera similar en todas las ocupaciones. No obstante, se detectan diferencias entre los ejemplares arqueológicos y los modernos. Los ejemplares de Túnel VII presentan un índice medio más bajo (valvas más bajas), lo que puede relacionarse o con una recolecta en las franjas más profundas del eulitoral (medio/inferior) donde es más fácil mantener el grado de hidratación necesario o en zonas más expuestas a la acción del oleaje (Morriconi, 2005).

La explicación más plausible en la que coinciden los resultados obtenidos de las dos especies es que estas se hayan obtenido en zonas favorables a su desarrollo que, según los datos actuales (Morriconi \& Calvo, 1993; Morriconi, 2005), podría corresponder con una explotación mayoritaria de la franja media e inferior del eulitoral, donde las condiciones son más estables (menor fuerza de las olas e hidratación constante).

Los datos obtenidos de Mytilus edulis presentan el mismo patrón que $N$. magellanica. No hay diferencias significativas entre las 
ocupaciones de Túnel VII, lo que sugiere una explotación de zonas con similares características en las distintas ocupaciones del asentamiento. Sin embargo, sí existen diferencias significativas entre las poblaciones moderna y arqueológica. Los ejemplares de Túnel VII presentan índices más elevados que los ejemplares actuales, lo que significa que son más robustos (Fig. 4). Según los estudios de poblaciones actuales, estos datos se pueden interpretar como la consecuencia de soportar condiciones más desfavorables que los individuos actuales (por ejemplo, cambios de temperatura, salinidad e insolación) (Bala, 1989; Silva \& Calvo, 1995; Silva, 1996). Estos datos podrían relacionarse con algunos estudios paleoambientales sobre el Holoceno reciente de Tierra del Fuego que también sugieren un empeoramiento de las condiciones climáticas para un periodo breve comprendido entre 1800 y 1930 (Mauquoy et al. 2004), correspondiente con el momento de ocupación de Túnel VII. Para ese periodo se detecta un aumento del frío y la humedad (Mauquoy et al. 2004). Según el trabajo de Obelic et al. (1998) la temperatura obtenida de ejemplares de sitios correspondientes al s. XIX es $0,8^{\circ} \mathrm{C}$ inferior a la media actual obtenida en el canal Beagle $\left(6,5^{\circ} \mathrm{C}\right)$.

En el caso de Lanashuaia, los test de varianza indican que existen diferencias significativas entre los indices obtenidos de los ejemplares arqueológicos de N. deaurata y N. magellanica y los modernos, obtenidos en la bahía Cambaceres exterior. Los individuos arqueológicos de las dos especies presentan un índice medio significativamente más bajo que el obtenido de los ejemplares actuales de la zona superior/media del eulitoral. Las valvas proporcionalmente más bajas de $N$. deaurata indican que provienen de zonas con poca acción del oleaje y para $N$. magellanica, indicarían una procedencia de zonas que permiten su hidratación (Morriconi \& Calvo, 1993; Morriconi, 2005). Igual que en Túnel VII, podría indicar una mayor explotación de las zonas inferiores del intermareal, siempre cubiertas por el agua. Las dos especies presentan poca variabilidad en los datos, lo que correspondería con una explotación de zonas similares a lo largo de toda la ocupación del sitio.

La población de Mytilus edulis de Lanashuaia presenta individuos más robustos que los obtenidos actualmente en las bahías Cambaceres interior y exterior (Bala, 1989; Silva \& Calvo, 1995; Silva, 1996). La bahía Cambaceres interior es más resguardada y calma que la exterior, lo que se refleja en la forma más estilizada de los mejillones (indices bajos) (Fig. 5). El gráfico de cajas indica que los ejemplares de Lanashuaia son similares a la población de la Cambaceres exterior, por lo que se podría sugerir una recolección predominante en esta bahía, aunque el test de varianza indica que existen diferencias significativas entre las dos poblaciones. Según los estudios en poblaciones actuales del canal Beagle (Silva y Calvo, 1995; Silva, 1996) y de otras zonas (Seed, 1968), la mayor robustez de los mejillones de Lanashuaia podría indicar condiciones más desfavorables que las que la costa presenta en la actualidad, coincidiendo con lo documentado en Túnel VII y con la información paleoambiental, que apunta a un empeoramiento en las condiciones ambientales para este periodo (Obelic et al. 1998; Mauquoy et al. 2004).

\section{CONCLUSIONES}

Túnel VII y Lanashuaia corresponden cronológicamente al momento de contacto con los colonos europeos (s. XVIII-XIX). Los estudios morfométricos en las especies de moluscos mayoritarias (Nacella deaurata, Nacella magellanica y Mytilus edulis) y la comparación con ejemplares actuales sugieren en los dos sitios una explotación similar de las costas. En los dos yacimientos los datos obtenidos de las lapas (Nacella deaurata y Nacella magellanica) sugieren una explotación de todo el gradiente intermareal. Estos datos coinciden con la información biométrica que indica una explotación intensiva de estos taxones, pero sin llegar a su sobreexplotación (VerdúnCastelló, 2014b).

Los mejillones (Mytilus edulis) presentan en los dos yacimientos morfologías más robustas que las detectadas actualmente en las costas cercanas. Esta mayor robustez de los mejillones puede ser debida a condiciones más desfavorables que las actuales. Tal como apuntan algunos estudios paleoambientales, se ha documentado un aumento del frío y la humedad para un periodo corto de tiempo correspondiente con el momento de ocupación de los yacimientos (Obelic et al. 
1998; Mauquoy et al. 2004). No obstante, las condiciones locales tienen una influencia muy importante en la morfología de los moluscos (Seed, 1968, 1980; Gaspar et al. 2002) y posiblemente habría que buscar la explicación en estos factores. En Lanashuaia los datos morfológicos indican una recolección principalmente en la bahía Cambaceres exterior o en costas análogas, ya que los índices obtenidos del material arqueológico son muy similares a los actuales obtenidos en esa zona.

Las conclusiones presentadas por este estudio muestran que es posible relacionar las diferencias en la morfología de los moluscos con su recolección en distintos lugares, lo que puede ofrecer información sobre la zona de captación de estos recursos. No obstante, es necesario un estudio extenso sobre las costas actuales, las variaciones morfométricas de los moluscos que las habitan y los factores locales que pueden afectar a su morfología para tener una base de datos comparativa exhaustiva. Igualmente se ha demostrado que la aplicación de los análisis morfométricos en el estudio de las sociedades que explotaban los recursos costeros es una fuente de información importante sobre su gestión y área de captación.

\section{AGRADECIMIENTOS}

Agradezco a André C. Colonese los comentarios sobre la primera versión de este trabajo y las sugerencias y comentarios de dos revisores/as anónimos que contribuyeron a mejorarlo notablemente. También agradezco a las personas que de manera desinteresada colaboraron en la recolección de los ejemplares de moluscos modernos (Bernabé Urtubey, Débora Zurro, Ivan Briz, Mauricio González y Myrian Álvarez).

\section{BIBLIOGRAFÍA}

Álvarez, M., Zurro, D., Briz, I., Madella, M., Osterrieth, M. \& Borrelli, N. (2009). Análisis de los procesos productivos en las sociedades cazadoras-recolectoras-pescadoras de la costa norte del Canal Beagle (Argentina): el sitio Lanashuaia. En M. Salemme, F. Santiago, M. Álvarez, E. L. Piana, M. Vázquez y M. E. Mansur (Comps.), Arqueología de la Patagonia. Una mirada desde el último confín (pp. 903-918). Ushuaia, Ed. Utopías.

Bala, L. O. (1989). Biología y ecología del mejillón (Mytilus edulis platensis) en el golfo San José, provincia del Chubut. Tesis doctoral, Universidad Nacional de La Plata, Facultad de Ciencias Naturales y Museo.

Behrens Y., S. \& Peters, E. E. (1988). Harvest management and the growth and condition of submarket-size sea mussels, Mytilus californianus. Aquaculture, 74, 293-299

Bridges, L. (1975) [1948]. El último confín de la Tierra. Buenos Aires: Ed. Marymar.

Briz, I., Álvarez, M., Zurro, D. \& Caro, J. (2009). Meet for lunch in Tierra del Fuego: a new ethnoarchaeological project. Antiquity, Project Gallery, 83(322). http:// www.antiquity.ac.uk/projgall/briz322/

Cabral, J. P. \& da Silva, A. C. F. (2003). Morphomeric analysis of limpets from an Iron-Age shell midden found in nothwest Portugal. Journal of Archaeological Science, 30, 817-829.

Campbell, G. (2008). Beyond means to meaning: using distributions of shell shapes to reconstruct past collecting strategies. Environmental Archaeology, 13(2), 111-121.

Campbell, G. (2014). Size prediction in archaeomalacology: the common mussel, Mytilus edulis L., as an example. Archaeological and Anthropological Sciences, 6, 305-318.

Claassen, C. (1998). Shells. Cambridge: University Press.

Cabral, J. P. (2010). O depósito de conchas do sítio arqueológico das "Areias Altas" (Porto, Portugal). Estudo morfológico e morfométrico das conchas inteiras de moluscos. Férvedes, 6, 73-82.

Colonese, A. C., Camarós, E. Verdún, E., Estévez, J., Giralt, S. \& Rejas, M. (2011). Integrated archaeozoological research of shell middens: new insights into huntergatherer-fisher coastal exploitation in Tierra del Fuego. Journal of Island and Coastal Archaeoloy, 6, 235254.

Gaspar, M. B., Santos, M. N., Vasconcelos, P. \& Monteiro, C. C. (2002). Shell morphometric relationships of the most common bivalve species (Mollusca: Bivalvia) of the Algarve coast (southern Portugal). Hydrobiologia, 477, 73-80

Estévez, J. \& Vila, A. (1995) (coord.). Encuentros en los conchales fueguinos, Treballs d'etnoarqueologia. Universitat Autònoma de Barcelona, Departament d'Antropologia Social i Prehistòria, CSIC, Madrid.

Estévez, J. \& Vila, A. (2006). Variability in the lithic and faunal record through 10 reoccupations of a XIX century Yamana Hut. Journal of Anthropological Archaeology, 25, 408-423. 
Gusinde, M. (1986) [1937]. Los indios de Tierra del Fuego. Los Yámana. Buenos Aires: Centro Argentino de Etnología Americana, Consejo Nacional de Investigaciones Científicas y Técnicas.

Hallmann, N., Burchell, M., Schöne, B. R., Irvine, G. V. y Maxwell, D. (2009). High-resolution sclerochronological analysis of the bivalve mollusk Saxidomus gigantea from Alaska and British Columbia: techniques for revealing environmental archives and archaeological seasonality. Journal of Archaeological Science, 36, 2353-2364.

Hammer, Ø., Harper, D. A. T. \& Ryan, P. D. (2001). PAST: Paleontological Statistics Software Package for Education and Data Analysis. Palaeontologia Electronica, 4(1). http://palaeo-electronica. org/2001_1/past/issue1_01.htm

Hyades, P. D. \& Deniker, J. (1891). Anthropologie et Ethnographie. Mission Scientifique du Cap Horn (1882-1883), vol. VII, París.

Mauquoy, D., Blaauw, M., van Geel, B., Borromei, A., Quattrocchio, M., Chambers, F. M. \& Possnerte, G. (2004). Late Holocene climatic changes in Tierra del Fuego based on multiproxy analyses of peat deposits. Quaternary Research, 61, 148-158.

Malanga, G.; Estévez, M. S.; Calvo, J.; Abele, D. \& Puntarulo, S. (2005). Oxidative stress in gills of limpets from the Beagle Channel: comparison with limpets from the Antarctic. En W. E. Arntz, G. A. Lovrich y Thatje, S. (Eds.) Scientia Marina. The Magellan-anctartic connection: links and frontiers at high southern latitudes, 69 (Suppl. 2), 297-304.

Mameli, L. \& Estévez, J. (2004). Etnoarqueología de aves: el ejemplo del extremo sur americano, Treballs d'Etnoarqueologia. Madrid, CSIC 5.

Mason, R. D., Peterson, L. \& Tiffany, J. A. (1998). Weighing vs. Counting: measurement reliability and the California School of Midden Analysis. American Antiquity, 63(2), 303-324.

Morriconi, E. \& Calvo, J. (1993). Influencia ambiental sobre el crecimiento alométrico de la valva en Nacella (Patinigera) deaurata (Gmelin, 1791) del Canal Beagle, Argentina. Malacologia, 35(1), 135-140.

Morriconi, E. (1999). Reproductive biology of the limpet Nacella (P.) deaurata (Gmelin, 1791) in Bahía Lapataia (Beagle Channel). Scientia Marina, Magellan-Antarctic: ecosystems that drifted apart, 63 (Supl. 1), 417-426.

Morriconi, E. (2005). Ecología reproductiva de Moluscos Gasterópodos en el Canal Beagle. Tesis doctoral, Universidad Nacional de La Plata, Facultad de Ciencias
Naturales y Museo.

Obelic, B., Álvarez, A., Argullós, J. \& Piana, E. L. (1998). Determination of water palaeotemperature in the Beagle Channel (Argentina) during the last $6000 \mathrm{yr}$ though stable isotope composition of Mytilus edulis shells. Quaternary of South America and Antarctic Peninsula, 11, 47-71.

Orquera, L. A. (1999). El consumo de moluscos por los canoeros del extremo sur. Relaciones de la Sociedad Argentina de Antropología, XXIV, 307-327.

Orquera, L. A. (2005). Mid-Holocene littoral adaptation at the southern end of South America. Quaternary International, 132, 107-115.

Orquera, L. A. \& Piana E. L. (1992). Un paso hacia la resolución del palimpsesto. En L. A., Borrero y J. L. Lanata (Comps.), Análisis espacial en la arqueología patagónica (pp. 21-52). Buenos Aires, Ed. Ayllu SRL.

Orquera, L. A. \& Piana E. L. (2000). Composición de los conchales de la costa del Canal Beagle (Tierra del Fuego, República Argentina)- primera parte. Relaciones de la Sociedad Argentina de Antropología, XXV, 249-274.

Orquera, L. A. \& Piana E. L. (2009). Sea nomads of the Beagle Channel in Southernmost South America: over six thousand years of coastal adaptation and stability. Journal of Island \& Coastal Archaeology, 4, 61-81.

Piana, E. L., Estévez, J. \& Vila., A. (2000). Lanashuaia: un sitio de canoeros del siglo pasado en la costa norte del canal Beagle. En En el País de los Gigantes. Perspectivas arqueológicas en Patagonia, Actas de las Cuartas Jornadas de Arqueología de la Patagonia, Río Gallegos- 2 a 6 de noviembre de 1998 (pp. 455469). Río Gallegos, Universidad Nacional de Patagonia Austral, Unidad Académica de Río Gallegos, II.

Piana, E. L. \& Orquera, L. A. (2010). Shellmidden formation at the Beagle Channel (Tierra del Fuego, Argentine). En D. Calado, M. Baldia y M. Boulanger (Eds.), Monumental Questions: Prehistoric Megaliths, Mounds, and Enclosures. Proceedings of the XV World Congress (Lisbon, 4-9 September 2006) (pp. 263-271). Oxford, BAR International Series, Archaeopress, vol. 7.

Rick, T. C., Kennett, D. J. \& Erlandson, J. M. (2005). Preliminary report on the archaeology and paleoecology of the Abalone Rocks Estuary, Santa Rosa Island, California. En D. Garcelon y Schwemm, C. (Eds.), Proceedings of the Sixth California Islands Symposium, (pp. 55-63). California, National Park Service Technical Publication, Institute for Wildlife Studies.

Russell, G. (1991). Vertical distributions. En A. C. Mathieson y P. H. Nienhuis (Eds.), Ecosystems of the world. 
Intertidal and littoral ecosystems, (pp. 43-66). Elsevier, 24.

Seed, R. (1968). Factors influencing shell shape in the mussel Mytilus edulis. Journal of Marine Biological Assotiation of United Kingdom, 48, 561-584.

Seed, R. (1980). Shell growth and form in the bivalvia. En D. C. Rhoads y R. A. Lutz (Eds.), Skeletal growth of aquatic organisms. Biological records of environmental change (pp. 23-67). New York and London: Plenum press.

Silva, Mar. R. (1996). Patrón de asentamiento larval y crecimiento de Mytilus edulis chilensis en el Canal Beagle. Provincia de Tierra del Fuego, Antártida e Islas del Atlántico Sur, Secretaría de Planeamiento, Ciencia y Tecnología, Dirección de Ciencia y Tecnología, informe inédito.

Silva, Ma . R. \& Calvo, J. (1995). Influencia ambiental sobre la morfología valvar de Mytilus edulis chilensis del Canal Beagle. En Actas del IV Congreso Latinoamericano de Ciencias del Mar, Mar del Plata, 23-27 de octubre, 1995: 186.

Verdún C., E. (2006). Análisis de la malacofauna de un conchero.
El ejemplo de Túnel VII (Tierra del Fuego, Argentina. Revista Atlántico Mediterránea de Prehistoria y Arqueología Social, 8, 53-69.

Verdún C., E. (2010). Molluscs as sedimentary components. Another perspective of analysis. Munibe, Suplemento, 31, 294-301.

Verdún C., E. (2014a). El consumo de moluscos en sociedades cazadoras-recolectoras de Tierra del Fuego (Argentina). En .J. Oría y A. M. Tívoli (eds.) Cazadores de mar y tierra. Estudios recientes en arqueología fueguina (pp. 363-387). Ushuaia, Ed. Cultural de Tierra del Fuego, Museo del Fin del Mundo.

Verdún C., E. (2014b). Application of biometric analyses on shell middens of hunter-fisher-gatherer societies of Tierra del Fuego (Argentine). Archaeofauna. International Journal of Archaeozoology. Proceedings of the II Reunión Científica de Arqueomalacología de la P. Ibérica, UAB 19-20 mayo 2011, 23, 221-236.

Zaixso, H. E., Boraso, A. L. \& López Gappa, J. J. (1978). Observaciones sobre el mesolitoral rocoso de la zona de Ushuaia (Tierra del Fuego, Argentina). Ecosur, 5(10), 119-130. 